\title{
A comparative assessment of marker expression between cardiomyocyte differentiation of human induced pluripotent stem cells and the developing pig heart
}

\author{
Lauschke, Karin; Volpini, Luca; Liu, Yong; Vinggaard, Anne Marie; Hall, Vanessa J
}

Published in:

Stem Cells and Development

Link to article, DOI:

$10.1089 /$ scd.2020.0184

Publication date:

2021

Document Version

Peer reviewed version

Link back to DTU Orbit

Citation (APA):

Lauschke, K., Volpini, L., Liu, Y., Vinggaard, A. M., \& Hall, V. J. (2021). A comparative assessment of marker expression between cardiomyocyte differentiation of human induced pluripotent stem cells and the developing pig heart. Stem Cells and Development, 30(7), 374-385. https://doi.org/10.1089/scd.2020.0184

\section{General rights}

Copyright and moral rights for the publications made accessible in the public portal are retained by the authors and/or other copyright owners and it is a condition of accessing publications that users recognise and abide by the legal requirements associated with these rights.

- Users may download and print one copy of any publication from the public portal for the purpose of private study or research.

- You may not further distribute the material or use it for any profit-making activity or commercial gain

- You may freely distribute the URL identifying the publication in the public portal 


\section{A comparative assessment of marker expression between cardiomyocyte differentiation of human iPSCs and the}

\section{developing pig heart}

Karin Lauschke ${ }^{1,2,+, *}$, Luca Volpini ${ }^{3, *}$, Yong Liu ${ }^{3}$, Anne Marie Vinggaard ${ }^{1}$ and Vanessa Jane Hall ${ }^{3}$

1: National Food Institute, Technical University of Denmark, Kemitorvet, 2800 Kongens Lyngby, Denmark

2: Department for Biotechnology and Biomedicine, Technical University of Denmark, Produktionstorvet, 2800 Kongens

Lyngby, Denmark

3: Department of Veterinary and Animal Sciences, University of Copenhagen, Dyrlægevej 100, 1870 Frederiksberg,

Denmark

+ corresponding author: karla@food.dtu.dk

* authors contributed equally to this work

Email addresses:

Luca Volpini: Ivolpini87@gmail.com

Yong Liu: plq199@alumni.ku.dk

Anne Marie Vinggaard: annv@food.dtu.dk

Vanessa Jane Hall: vh@sund.ku.dk

Running title: Cardiac markers in hiPSC and developing pig heart

Published at Stem Cells and Development:"

Published Online: 18 Feb 2021 https://doi-org.proxy.findit.dtu.dk/10.1089/scd.2020.0184 


\section{Abstract}

1 The course of differentiation of pluripotent stem cells into cardiomyocytes and the intermediate cell types are

2 characterized using molecular markers for different stages of development. These markers have been selected

3 primarily from studies in the mouse and from a limited number of human studies. However, it is not clear how

4 well mouse cardiogenesis compares with human cardiogenesis at the molecular level. We tackle this issue by

5 analyzing and comparing the expression of common cardiomyogenesis markers (PDGFR- $\alpha$, FLK1, ISL1,

6 NKX2.5, CTNT, CX43 and MYHC-B) in the developing pig heart at embryonic day (E)15, E16, E18, E20, E22

7 and E24 and in differentiating cardiomyocytes from human induced pluripotent stem cells (hiPSC). We found

8 that porcine expression of the mesoderm marker FLK1 and the cardiac progenitor marker ISL1 was in line with

9 our differentiating hiPSC and reported murine expression. The cardiac lineage marker NKX2.5 was expressed

10 at almost all stages in the pig and human IPSC, with an earlier onset in the hiPSC compared to reported murine

11 expression. Markers of immature cardiomyocytes, CTNT and MYHC-B were consistently expressed

12 throughout E16 - E70 in the pig, which is comparable with mouse development, whereas the markers

13 increased over time in the hiPSC. However, the commonly used mature cardiomyocyte marker, CX43, should

14 be used with caution, as it was also expressed in the pig mesoderm, as well as hiPSC immature

15 cardiomyocytes, whilst this has not been reported in mice. Based on our observations in the various species,

16 we suggest to use FLK1/PDGFR- $\alpha$ for identifying cardiac mesoderm, ISL1/NKX2.5 for cardiac progenitors and

17 CTNT/MYHC-B for immature cardiomyocytes. Further, CTNT+/ISL1+ could mark immature cardiomyocytes

18 and CTNT+/ISL1- mature cardiomyocytes. CX43 should be used together with sarcomeric proteins. This

19 knowledge may help improving differentiation of hiPSC into more in vivo like cardiac tissue in the future.

22 Keywords: Heart development, embryoid body, cardiac marker, pig, induced pluripotent 23 stem cell, cardiomyocyte differentiation 
28 Replicating heart development in vitro using pluripotent stem cells (PSCs) is dependent on a series of 29 developmental changes in a short time frame that is verified using well-established cardiomyocyte markers.

30 As PSCs are guided through cardiomyogenesis, they undergo developmental changes similar to those in the 31 developing embryo, including mesoderm formation, marked by expression of T Brachyury (BRACHYURY) and 32 cardiac mesoderm marked by posterior BHLH transcription factor (MESP1), Fetal liver kinase 1 (FLK1) and 33 Platelet-Derived Growth Factor Receptor, Alpha Polypeptide (PDGFR- $\alpha$ ). These cells then form cardiac 34 progenitors that express insulin gene enhancer protein (ISL1), the NK2 homeobox 5 (NKX2.5), GATA binding 35 protein 4 (GATA4) and T-Box transcription factor 5 (TBX5), before they differentiate into immature 36 cardiomyocytes marked by cardiac troponin T (CTNT), Myosin Heavy chain 7 (MYHC-B) and other cell surface 37 markers [1]. Maturation of cardiomyocytes takes several weeks and is more complex to assess, as not only 38 markers of gap junctions such as gap junction protein Connexin43 (CX43) are used to characterize maturation 39 [2-4], but also electrophysiological methods, calcium profiles, myofibril organization and sarcomere striation

40 [1]. These gene markers are generally accepted in the field and have been identified from mouse (and to a 41 much lesser extent from human) cardiac development studies and are applied to human stem cell 42 differentiation studies to identify the different stages of cardiac differentiation [1,5-10]. To our knowledge, only 43 three studies have investigated gene expression in human fetal hearts [11-13] and comparisons to mouse 44 heart development indicate that differences exist. For example, MYHC-B is upregulated in human ventricular 45 cardiomyocytes while it is downregulated in these cells in mice [14]. Further, different mesoderm populations 46 exist in the human which form for e.g. CD235A $A^{+}$cardiomyocytes of the left ventricle, which have not been 47 identified in the developing mouse heart [15]. Another study on human PSC-derived cardiomyocyte-like cells 48 describes a novel marker for cardiac progenitor cells, LGR5, that is unique to human cardiac progenitor cells 49 [11]. It is also not clear if the markers used for cardiac differentiation studies are especially accurate for human 50 tissues. Unfortunately, investigation of human heart development remains limited due to the rarity of available 51 tissue to researchers.

53 Identification of alternative animal models of heart development, which better replicate the molecular 54 mechanisms driving human cardiac development would be an advantage for the field. The pig is a strong 55 candidate, since pig embryos develop as bilaminar embryonic discs (flat disc) just like human embryos [16]. 
Developing pig embryos and fetuses are easily obtained through pig production farms and are a good alternative to using non-human primate embryos/fetuses for studying development [17-21]. A detailed molecular characterization of early pig heart development is absent from the literature. Only limited knowledge is available on the prenatal developing heart $[22,23]$ and one study was performed on the postnatal developing heart [24]. Molecular profiling has recently been performed on the adult pig heart, which shows clear commonalities with the mouse heart, relating to metabolic processes such as carbohydrate metabolism. Unique proteins were also expressed in the pig heart although not clearly depicted in the article [25]. Here, we provide the first molecular studies of cardiogenesis in the pig and cross-compare it to an established human PSC-based cardiac differentiation protocol [26], which results in almost $100 \%$ contracting cardiac spheres 65 after seven days.

The aim of this study was to determine whether commonly used markers of early heart development were aligned between the pig and differentiating human iPSC cardiomyocytes and to assess whether there were differences to published mouse studies. We provide detailed anatomical and molecular insight into the developing pig heart, with focus on the most commonly used markers PDGFR- $\alpha$, FLK1 (cardiac mesoderm), ISL1, NKX2.5, (cardiac progenitors) MYHC-B, CTNT (immature cardiomyocytes) and CX43 (mature cardiomyocytes) in an attempt to assess whether refinement of the use of molecular markers used in human cardiac differentiation studies is needed.

\section{2. Materials and methods}

\subsection{Pigs embryos and fetal pig heart}

77 Pig embryos were obtained at day embryonic day (E)15, E16, E18, E20, E22 and E24 of gestation (three 78 embryos per day) following insemination of Landrace/Yorkshire sows (twice within $24 \mathrm{~h}$ ) using semen from a 79 Duroc boar from a local pig production farm (Roskilde, Denmark). The sows were slaughtered at the above predefined days calculated after the second insemination and the embryos were collected within 30 min after

81 slaughter and transported at $37^{\circ} \mathrm{C}$ to the University of Copenhagen, where the fetuses (clinically dead from

82 asphyxiation during transport) were isolated by umbilical excision from their fetal sacs. A pig heart from E70

83 was also dissected from a fetus. The embryos and heart were fixed in $4 \%$ paraformaldehyde (PFA) in PBS at

$844^{\circ} \mathrm{C}$ overnight and stored until use in PBS at $4^{\circ} \mathrm{C}$ containing $0.02 \%$ sodium azide. 


\subsection{Tissue preparation and Haematoxylin and Eosin staining}

86 The embryos and heart were paraffin embedded in a tissue processor (Thermo Fisher Scientific, Waltham,

87 USA), where they were dehydrated using an increasing ethanol concentration and then "cleared" in Estisol220

88 before infiltration with paraffin. Subsequently, the specimens were embedded in paraffin wax (Paraffin wax

89 blended with synthetic polymers, VWR, Radnor, USA). From the paraffin blocks sagittal sections (5 $\mu \mathrm{m})$ were

90 cut using a microtome (SM2000R, Leica, Wetzlar, Germany). The sections were mounted onto glass slides

91 and stored at $4{ }^{\circ} \mathrm{C}$. For initial histological examination of the tissue, sections were deparaffinized, rehydrated

92 and every $5^{\text {th }}$ or $10^{\text {th }}$ section (depending on embryo size) was stained with haematoxylin-eosin.

\subsection{Immunohistochemistry}

94 Paraffin sections were heated at $60^{\circ} \mathrm{C}$ for $40 \mathrm{~min}$ and rehydrated through xylene and ethanol washing. Slides

95 were dipped twice for 10 min each in xylene (VWR) followed by decreasing ethanol concentrations : twice 5

96 min in $99 \%$ ethanol, 3 min in $96 \%$ ethanol and 3 min in $70 \%$ ethanol. Immunohistochemistry was performed

97 using two different protocols depending on the species of the antibody used. Primary antibodies: FLK-1 Rabbit

98 polyclonal IgG (1:50, Abcam, Ab45010), NKX2-5 Rabbit polyclonal IgG (1:25, Thermo Fisher Scientific, PA5-

99 49431), ISL1 Rabbit polyclonal IgG (1:300, Merck Millipore, AB4326), CX43 Rabbit polyclonal IgG (1:1000,

100 Abcam, Ab11370), CTNT Mouse monoclonal IgG2b (1:400, Abcam, Ab10214), MYHC-B Mouse monoclonal

$101 \lg 1$ (1:4000, Abcam, Ab11083). The following negative controls were used:Negative Mouse IgG1 (1:40,

102 DAKO, x0931), Normal Rabbit IgG (1:2000, DAKO, x0936), Negative Mouse IgG2b (1:40, DAKO, x0944).

103 These antibodies were isolated from non-immunized animals and are well suited to identify non-specific

104 binding to cell surface components. The following secondary antibodies were used: Secondary Antibody

105 Biotinylated goat anti-rabbit (1:250, DAKO, E0432), Third Layer Streptavidin/HRP (1:500, DAKO, P0397, lot.

$10600032671,0.83 \mathrm{~g} / \mathrm{L})$. For monoclonal mouse antibodies we used the UltraVision ${ }^{\mathrm{TM}}{ }^{\mathrm{Q}}$ Quanto Detection System

107 HRP DAB (Thermo Fisher Scientific). Briefly, rehydrated sections were washed for 5 min in Milli-Q water (MQ)

108 and antigen retrieval was performed by immersion three times $5 \mathrm{~min}$ in boiling $0.01 \mathrm{M}$ sodium citrate solution

109 (PH 6, Merck Millipore, Burlington, USA). Sections were then washed in $4{ }^{\circ} \mathrm{C}$ PBS and placed on ice. The

110 Ultravision Hydrogen Peroxide Block was then applied for $10 \mathrm{~min}$, followed by washing in PBS for 5 min and

111 incubation in the Block for another 5 min. Protein block was decanted prior to a 30 min incubation with primary

112 antibodies. Again the sections were washed in PBS for 5 min and incubated with Primary Antibody Amplifier

113 Quanto for $10 \mathrm{~min}$ followed by another PBS wash for $5 \mathrm{~min}$. In the last step, the sections were washed $3 \mathrm{~min}$ 
114 in PBS, then 3 min in MQ and 3 min in PBS. The sections were then stained with DAB+ Substrate Chromogen

115 system (DAKO, Glostrup, Denmark) for $5 \mathrm{~min}$. For the polyclonal rabbit antibody, sections were kept $5 \mathrm{~min}$ in

116 PBS following rehydration and permeabilized with $0.1 \%$ TritonX-100 (Merck Millipore) for 30 min. The sections

117 were washed twice for $5 \mathrm{~min}$ in PBS prior to antigen retrieval (performed as above). The sections were

118 subsequently incubated with $3 \% \mathrm{H}_{2} \mathrm{O}_{2}$ in water for $10 \mathrm{~min}$. After two washes in PBS for $5 \mathrm{~min}$, the slides were

119 blocked with $10 \%$ Normal Goat Serum (NGS, DAKO) in $1 \%$ Bovine Serum Albumin (blocking buffer) (BSA,

120 Merck Millipore) in PBS for $1 \mathrm{~h}$, washed shortly in PBS and then incubated with primary antibody diluted in

121 blocking buffer overnight at $4{ }^{\circ} \mathrm{C}$. The next day, after two washes for $5 \mathrm{~min}$ in PBS, the sections were incubated

122 in secondary antibody diluted in blocking buffer for $30 \mathrm{~min}$, washed again twice for $5 \mathrm{~min}$ in PBS and then

123 incubated in Streptavidin/HRP diluted in blocking buffer for $30 \mathrm{~min}$. After two washes in PBS for 5 min, the

124 slides were visualized using one drop DAB Chromogen per $\mathrm{mL}$ substrate buffer (DAKO) for $5 \mathrm{~min}$. In the final

125 steps of both protocols, the sections were washed in MQ water for $5 \mathrm{~min}$, counterstained using $0.5 \%$ methyl

126 green (Sigma-Aldrich, St. Louis, USA) in $0.1 \mathrm{M}$ Sodium Acetate Buffer for $2 \mathrm{~min}$, washed twice for 3 min in MQ

127 water, dipped 10 times in $96 \%$ ethanol, submerged in xylene and mounted with Distyrene Plasticizer Xylene

128 (DPX, Merck Millipore). Images were captured using a Leica DFC490 digital camera and a Leica DMR

129 microscope and Leica application suite software.

\section{6 hiPSC culture}

131 For experiments with human cells, the hiPSC line Bioni010-C (Bioneer A/S, Hoersholm, Denmark) was used.

132 Cells were cultured in mTeSR ${ }^{\mathrm{TM}} 1$ serum-free medium ( STEMCELL Technologies, Vancouver, Canada) in 6

$133 \mathrm{~cm}$ cell culture dishes (Nunclon ${ }^{\mathrm{TM}}$ Delta Surface, Thermo Fisher Scientific) coated with hESC-Qualified

134 Matrigel (354277, Corning, Corning, USA). The medium was exchanged daily and cells were split weekly with

135 EDTA. Briefly, medium was removed from the dish, the cells washed once with DPBS (Dulbecco's Phosphate

136 Buffered Saline, Sigma-Aldrich) and $1 \mathrm{ml} 0.02 \%$ EDTA in DPBS added. The cells were incubated for 1-3 min

137 and the EDTA was removed. The cells were harvested in small clumps in $\mathrm{mTeSR}{ }^{\mathrm{TM}} 1$ and transferred to a new

138 plate according to the split ratio. The hiPSCs were incubated at $37^{\circ} \mathrm{C}$ and $5 \% \mathrm{CO}_{2}$.

\subsection{Embroid body formation and cardiac differentiation}

$1403 \mathrm{D}$ cultures of embryoid bodies (EBs) that mimic the early human blastocyst were generated from hiPSCs and

141 subsequently differentiated into cardiomyocytes as described in [26]. For harvesting hiPSCs, medium was

142 removed from the culture dish and $1 \mathrm{ml} \mathrm{Gibco}^{\text {TM }}$ TrypLE $^{\mathrm{TM}}$ Select (Thermo Fisher Scientific) added and 
143 incubated for 1-2 min. TrypLE was removed and the cells detached from the plate by adding $10 \mathrm{ml}$ mTeSR-

144 ROCK (mTeSR1 with Y27632 dihydrochloride). A single cell suspension was generated by pipetting several 145 times. The cells were counted and diluted to $5 \times 10^{4}$ cells $/ \mathrm{ml}$, of which $100 \mu \mathrm{l}$ per well were seeded into a 96146 Well Polystyrene Conical Bottom MicroWell ${ }^{\mathrm{TM}}$ Plate (249952, Thermo Fisher Scientific). The plates were 147 centrifuged at $500 \mathrm{~g}$ for $5 \mathrm{~min}$ and incubated at $37{ }^{\circ} \mathrm{C}$ and $5 \% \mathrm{CO}_{2} .20 \mathrm{~h}$ after seeding, and thereafter daily, 148 medium was exchanged by removing $80 \mu \mathrm{l}$ of medium and replaced with $80 \mu \mathrm{l}$ fresh medium according to this 149 schedule: day 0 (20 hr after seeding): day 0 medium, day 1: TS medium, day 2: Wnt medium, day 3: TS 150 medium, day 6: TS-medium (removal of $60 \mu \mathrm{l}$ per well and addition of $80 \mu \mathrm{l}$ per well on day 6). Media recipes 151 are given in supplementary material.

\subsection{Immunofluorescence of embryoid bodies}

153 The EBs were transferred from a culture plate to a $15 \mathrm{ml}$ centrifugation tube with a $1000 \mu \mathrm{L}$ pipette (wide orifice 154 tips). The spheres were left undisturbed for $5 \mathrm{~min}$ to allow gravity precipitation and all but approximately 0.5 $155 \mathrm{ml}$ supernatant was removed. Subsequently the spheres were resuspended and transferred to a $0.5 \mathrm{ml}$ tube.

156 For fixation, excess medium was removed and the spheres were washed once in PBS and fixed in $500 \mu \mathrm{l} 4 \%$ 157 formaldehyde for $15 \mathrm{~min}$ at room temperature (RT). The formaldehyde was removed and the spheres washed 158 three times in PBS and then stored in PBS at $4{ }^{\circ} \mathrm{C}$. Next spheres were permeabilized for $1 \mathrm{~h}$ at RT using 0.1 $159 \%$ Triton-X ( Merck Millipore) in PBS, washed $3 \mathrm{x}$ in PBS and incubated in blocking buffer (5 \% Normal donkey 160 serum (NDS) and $1 \%$ BSA in PBS) at RT for $2 \mathrm{~h}$. Primary antibodies (see section 2.3 and SSEA3 rat 161 (Biolegend 330302, 1:100), OCT4 goat (sc-8628, 1:100), SOX2 mouse (Millipore, SC1002, 1:1000)) diluted in 162 blocking buffer were incubated with the EBs on an orbital shaker at $4{ }^{\circ} \mathrm{C}$ overnight using gentle rotation. EBs 163 were washed in PBS for 10 min at RT, then incubated with secondary antibodies ( Donkey anti-rabbit: 164 ab150075 (AlexaFluor 647) Abcam (1:200), Donkey anti-mouse: ab150105 (AlexaFluor 488)Abcam (1:200), 165 Donkey anti-goat: ab150131 (AlexaFluor 647)Abcam (1:200)) in blocking buffer for $1 \mathrm{~h}$ at RT in the dark and 166 then washed again in PBS for 15 min at RT. For negative controls, D0 EBs were incubated without primary 167 antibody and only with secondary antibodies. For DNA labelling, EBs were incubated in Hoechst solution 10 $168 \mu \mathrm{g} / \mathrm{ml}($ Merck Millipore)) for $10 \mathrm{~min}$ in the dark. In the end, EBs were washed in PBS in the dark and immediately 169 prepared for mounting, thus transferred to glass slides and mounted with fluorescent mounting solution 170 (DAKO).

\subsection{Chromogen intensity quantification}


172 In order to create an overview of the expression data in the pig embryos, we estimated the expression levels

173 and classified them into four categories, which were then displayed in a heat map. For this, reciprocal intensity

174 of the chromogen stained immunohistochemistry sections was measured in order to quantify the amount of

175 marker protein present at the respective stages of pig heart development following the method by [27]. For

176 that, the images were opened in ImageJ and the area of interest selected with the elliptical selection tool

177 followed by the 'measure function' to retrieve the white area maximum. This value was subtracted from 250 ,

178 which is the maximum intensity value of white colour in a JPG image. The average reciprocal intensity for

179 every marker and developmental stage was calculated after measuring every image from three embryos. For

180 graphical illustration in a heat map, the ranges were converted into integer numbers according to Suppl. Table

181 2. With these values, a heat map was created using $R$ studio.

182

183 3. Results

1843.1 Anatomical characterization of pig heart development

185 There is little anatomical characterization of the developing prenatal pig heart, therefore, it was crucial to first

186 determine the timing of key anatomical stages of cardiac development. We decided to select embryos from

187 E15 - E24, since this is the range in which we presumed the heart begins to beat based on two reports $[22,23]$

188 as well as a later stage embryo from E70 to identify expression at a later midway stage during gestation

189 (gestation length in pig $=114$ days) (Fig. 1A). At E15 and E16, the pig cardiac tube was still smooth-walled but

190 clearly segmented into four dilatations, separated by indentations in a posterior-anterior orientation. The

191 expanded parts identifiable were the sinus venosus (SV) and the atrium (AT) (not yet enclosed within the

192 pericardial cavity), the ventricle (VE) and the bulbus cordis (BC) (Fig. 1A). Note, the truncus arteriosus is not

193 visible in these sections, since it is located on the right lateral side of the embryo. No circulation of red blood

194 cells could be observed at E15 or E16 of development. At E18, the cardiac tube was beginning to fold. It was

195 u-shaped and trabeculation was observable within the VE and BC. The AT was positioned above the VE and

196 enclosed in the pericardial cavity (Fig. 1A). We could also observe at this time point circulation of red blood

197 cells through the heart cavities. At E20, endocardial cushions and conotruncal cushions appeared, forming the

198 atrioventricular canal (AVC) and the aortic-pulmonary septum (Fig. 1A). At E22, the expansion of the primitive

199 left AT toward the truncus arteriosus was evident, and it was possible to observe the formed valves in the AVC 
and semilunar valves in the outflow tract (OFT). Furthermore, the formed trabeculation was visible in the VE myocardium wall, where the interventricular septum already appeared (Fig. 1A). At stage E24, the mitral valves

202 were observable in the AVC, as well as the semilunar valves in the OFT, regulating the blood flow, and the 203 atrium had surrounded the OFT (Fig. 1A). This anatomical description was important for defining the specific

204 timing of key events during early pig heart development, which are summarized in Fig. 1B and compared to 205 the same anatomical stages in mouse and human.

\subsection{Our in vitro cardiomyocyte differentiation model}

208 We applied an EB-based differentiation method to produce hiPSC-derived cardiomyocytes based on the use 209 of growth factors from the ActivinA/Nodal, BMP4 and Wnt pathways, which yields beating cardiomyocytes after 210 seven days [26]. The EBs on day (D)0 consisted of pluripotent cells as they expressed Oct3/4, Sox2 and 211 SSEA4 (Suppl. Fig. 1). The differentiation efficiency was very high as almost all EBs beat rigorously on D7, as 212 we showed before [26]. Since no maturation step was included, the cardiomyocytes on D7 represented 213 immature cardiomyocytes, as present in the embryo. Our cardiomyocyte differentiation model was therefore 214 in alignment with the developmental timeframe covered in our pig study, which corresponds to mouse E8.0 215 E11.5 and human gestational day $21-42$ (Fig. 1B).

217 3.3 Expression pattern of cardiac mesoderm and cardiac progenitor markers PDGFR- $\alpha, F L K 1$, ISL1 and 218 NKX2.5 in the developing pig heart and differentiating human iPSC

219 PDGFR- $\alpha$ and FLK1 together are well-established markers for cardiac mesoderm [28,29]. We investigated 220 only FLK1 expression in the developing pig heart, since two PDGFR- $\alpha$ antibodies (Santa Cruz sc-398206, 221 Abcam ab124392) we tested in our pig tissues failed to work (likely due to species-specific differences in the 222 antibody peptides). We found that FLK1 was expressed at the earliest time point studied, E15, in the pig but 223 became downregulated at later stages with the exception of expression observed in the endocardium cell 224 membrane and local blood vessels (Figs. 2 and 3). In the hiPSC cardiomyocytes, FLK1 was expressed on D1 225 and downregulated gradually thereafter, whereas PDGFR- $\alpha$ was expressed strongly for a longer period until 226 D6 (Fig. 4A).

227 In the case of cardiac progenitor marker expression, we observed ISL1 expression across all investigated time 228 points of the developing pig heart. It was expressed in the endocardium, myocardium and epicardium, as well 
as the first heart field (FHF: the first wave of mesodermal cells that differentiate to form the initial heart tube and that express muscle-specific proteins) and the second heart field (SHF) derivatives (Figs. 2 and 3). Of

231 note, during most stages, it was localized in the nucleus and cytoplasm, while at E70, ISL1 was only found in 232 the cytoplasm (Suppl. Fig. 2). Furthermore, ISL1 expression was not limited to the embryonic heart but also

233 expressed in other tissues, though not across the entire embryo, from E16 - E24 (Suppl. Fig. 3).

234 In the differentiating hiPSC, ISL1 was highly expressed across several time points from D1 to D6, but located

235 in the cytoplasm at D1-D2 and changed to nuclear localization at D3-D6 (Fig. 4A).

236 Expression of NKX2.5 in the developing pig heart was present from E16 to E70. Importantly, we detected

237 NKX2.5 in both the FHF and SHF derivatives, including the AT, AVC, VE and OFT. Moreover, we observed 238 expression throughout the endocardium, myocardium and epicardium (Figs. 2 and 3). There appeared to be 239 higher intensity on E18 and E22, however, this might be a technical artefact therefore we do not suggest these 240 peaks bear biological meaning (Figs. 2 and 3). In the human cardiomyocyte model, heterogenous expression 241 of NKX2.5 was observed in the D0 EBs and the expression increased thereafter. Interestingly, on D0 - D2 it 242 was mostly detected in the cells within the outer layer of the EB (Fig. 4A).

243 In order to ensure that our antibody stainings revealed true expression of the proteins, we performed extensive 244 antibody control stainings of the pig embryos and the EBs (as described in the Materials and Methods section). 245 No specific staining patterns were observed in the pig embryos and no signal was detected in the EBs (Suppl. 246 Fig. 4).

248 3.3. Comparative expression pattern of the immature cardiomyocyte markers CTNT, MYHC-B and mature 249 cardiomyocyte marker CX43 in the developing pig heart and human cardiomyocyte model

250 In the pig heart, CTNT was detectable as early as E16 specifically in the ventral looping tube of the pig heart 251 (Fig. 5). Expression was highest at E18, where it was found throughout the looping tube, as well as on E22, 252 leveling off thereafter (Figs. 3 and 5). Interestingly, in the human cardiomyocyte model, CTNT was detectable 253 at weak levels at early stages of development (D0 and D1) and became strongly expressed from D6 onwards 254 (Fig. 4B).

256 The mature cardiomyocyte marker CX43 was highly expressed at several phases of development in the pig 257 heart, including at E15, E20, E22, E24 and E70, whilst it was expressed at lower levels at E16 - E18 (Figs. 3, 
5 and Suppl. Fig. 2). Expression was localized to the myocardium of the AT, VE, BC and OFT (Fig. 5). In our human cardiomyocyte model, we found expression at all investigated stages. However, on D0 and D7 it appeared to be localized to gap junctions and from D1 - D6 localized in the cytoplasm (Fig. 4B).

262 In the pig heart, MYHC-B was expressed highly from E16 to E22 and decreased to low levels at E24 and E70 263 (Figs. 3, 5 and Suppl. Fig. 2). At E16, MYHC-B was located within the looping heart, and at the later stages 264 E18 - E24 in the myocardium of the AT, VE, BC and OFT (Fig. 5). At E70, MYHC-B was decreased within the 265 AT and limited to the mature ventricle (Suppl. Fig. 2). Interestingly, we observed a differential pattern of expression in the human cardiomyocyte model, namely expression of MYHC-B only at D7 (Fig. 4B).

\section{Discussion}

In this study, we characterized various stages of pig embryonic heart development using morphological features and molecular markers. We asked whether the markers used to identify different stages of human cardiomyocyte differentiation should be revised and determined if molecular differences exist between mouse 272 and pig cardiogenesis.

\subsection{Anatomical features of early development of the pig heart}

275 We report for the first time sequential anatomical events in early pig cardiac development, which recapitulate 276 early cardiac formation events in other mammalian species. At E15/E16 the pig heart is a linear tube and starts looping at E18. At E20 the endocardial cushions and conotruncal cushions appeared and were followed by

278 valve formation at E22. The outflow tract division became visible later at E24. Red blood cells were present from E18 onwards within the heart chambers suggesting the pig heart begins to pump later than E15, as previously reported [22]. Knowledge on this temporal timing of events will be useful for future studies investigating the early developing pig heart.

4.2 FLK1 is exclusively expressed in cardiac mesoderm but PDGFR- $\alpha$ is also expressed later on in cardiac progenitors

285 We found FLK1 to be a good mesodermal marker in the pig and that it was expressed early on in the differentiating hiPSCs as well. The expression of FLK1 in the pig is comparable to Flk1 expression in the 
mouse, which is observed in the broad mesoderm around the primitive streak at E7.5 [30] and declines towards the end of gestation [31]. Therefore, FLK1 appears to be conserved in expression between humans, pigs and mice. Contrary, PDGFR- $\alpha$ was expressed also at later stages in the differentiating human hiPSC, coexpressing with cardiac progenitor markers (ISL1 and NKX2.5) and therefore should only be used as a cardiac mesoderm marker when used together with FLK1.

\subsection{ISL1 is an excellent marker of cardiac progenitors but NKX2.5 is expressed throughout all stages}

294 In the pig, ISL1 was expressed from E15 through to E24. Although ISL1 is a transcription factor and therefore 295 expected to be detected only in the nucleus, we also found clear signals in the cytoplasm in the pig tissue as well as in the human EBs. Cytoplasmic expression of Isl1 has been previously shown in the mouse [33,34]. We speculate that cytoplasmic localization may regulate ISL1 function. In support of this, ISL1 was also detected in the cytoplasm at E70, a late stage where no ISL1 expression is expected. Lastly, we can also confirm that ISL1 is present in both heart fields as recently reported [35], disproving older studies of IsI1 as a second heart field marker [32]. In the differentiating hiPSC, ISL1 peaked on D2 in the nucleus, indicating the presence of cardiac progenitor cells at this stage of differentiation. With this, we can confirm with our study that ISL1 is a good marker of cardiac progenitors in large mammals.

Our finding that ISL1 was not only expressed in the heart but also in other tissues of the developing embryo overlaps with findings in the mouse. Here, Isl1 has been reported to be expressed during embryonic development in the pharyngeal mesoderm dorsal to the heart tube, the central nervous system, varying areas of the gastrointestinal tract, the hindlimbs, the umbilical cord and mesenchyme, the adrenal gland, thymus and

307 facial tissues. It has also been detected in a wide range of tissues in the adult in several studies $[36,37,46,38-$ 308 45].

309 In the case of NKX2.5 expression in the pig, we observed expression from E16 - E70 throughout the heart,

310 which is in accordance with Nkx2.5 expression in mice throughout gastrulation and adulthood [47] and confirms 311 more studies in mice that revoke an older notion of NKX2.5 as an FHF marker [35]. Therefore, we confirm that 312 NKX2.5 marks the progeny of both the FHF and SHF. In the hiPSC cardiomyocyte model we found that NKX2.5 313 was already expressed in a few cells from D0. This is in contrast to previous reports from mice, where NKX2.5 314 is expressed only in early heart progenitors [48] and at the cardiac progenitor stage in in vitro differentiation 315 protocols after induction of ISL1 [49,50]. We previously reported low levels of NKX2.5 gene expression at D0 
316 in the human EBs (four orders of magnitude lower than at D7) [26]. The expression we do observe in the in 317 vitro human iPSC model might reflect spontaneous induction of the cardiac lineage at a very early stage. Single 318 cell profiling of the EBs in the future might help confirm the genotype of these cells. Moreover, our 319 immunostaining results showed that on D0, D1 and D2, NKX2.5 was predominantly expressed in the outer 320 layer of the EBs, suggesting the presence of a differentiation wave, starting from the outside of the EB. Taken 321 together, NKX2.5 was expressed at almost all stages in the pig heart and human cardiomyocyte model, 322 supporting the notion that NKX2.5 is a good marker for the cardiac lineage that is conserved from mice to 323 humans.

4.4 CTNT is a good marker for immature cardiomyocytes and MYHC-B marks both cardiac progenitors and immature cardiomyocytes

327 We detected CTNT in the pig in a similar time window as reported in mice, namely in the linear heart tube at 328 E16 which corresponds to E8 in mice [51]. In the human cardiomyocyte model, however, it was detectable 329 very weakly at D0 and D1 before it became strongly expressed at D6 and D7. Interestingly, previous studies on in vitro cardiac differentiation only report CTNT expression at later stages when the cells begin to show 331 cardiomyocyte identity [2,52]. The weak expression of CTNT on D0 and D1 might indicate the presence of pre332 cardiac mesoderm, which has been shown to express CTnT in the mouse on day E7.5 prior to heart tube 333 formation [51]. In support of this, we have previously found that TNNT2 (the gene encoding CTNT) was 334 expressed in human iPSC at weak levels on D0, D1 and D2 of our differentiation, however, increasing by four 335 orders of magnitude at D7 [26]. When comparing the timely pattern of CTNT with other markers we found an 336 intriguing difference between the pig and the human model: While in the pig, CTNT and NKX2.5 were 337 upregulated together on E16 - 24, in the cardiomyocyte model CTNT was upregulated much later than 338 NKX2.5. Many other in vitro studies have reported the same relationship [53-56], while others have observed 339 a parallel regulation of CTNT and NKX2.5 as we detected in the pig [2,57]. This suggests that the human 340 differentiation protocols themselves affect the expression pattern of the developmental markers. Our findings 341 also suggest that the developmental pathways that take place in the developing heart are not replicated 342 identically to in vitro differentiation methods.

343 We found the immature cardiomyocyte marker MYHC-B to be expressed from E16 - E24 in the looping heart 344 and the myocardium of the pig and to be restricted to the ventricle at E70. Restriction to the ventricle has also 
been reported in human fetal hearts [58]. Of note, in rodents MYHC-B expression decreases postnataly and

346 is replaced by the variant MYHC-A [59]. Therefore, in contrast to humans, MYHC-A is the predominant form

347 in the adult ventricle in rodents [14]. In this study, we only analyzed MYHC-B as we expected the expression

348 in the pig to be more similar to humans than to rodents. Our data confirm this and show that MYHC-B is indeed

349 restricted to the ventricle at later stages of pig development. Furthermore, we conclude that MYHC-B also

350 marks cardiac progenitors and also specifically immature ventricular cardiomyocytes in the developing pig

351 heart. In differentiating human PSCs, we and others detected MYHC-B only at the later stages of differentiation

$352[2,3,60]$. This disagreement of MYHC-B levels in cardiac progenitors between in vitro and in vivo cardiac cells

353 has been shown before. MYHC-B was upregulated in fetal heart tissue compared to in vitro differentiated

354 cardiomyocytes at three weeks after first contraction [12] and in a recent RNA-Seq study where human fetal

355 heart progenitors from week 4 - 6 had 60-fold higher levels of MYHC-B expression than in vitro produced D3

356 progenitors [11]. This suggests that human PSC differentiation into cardiomyocytes may not faithfully replicate

357 the expression of MYHC-B in the human fetus. To sum, MYHC-B expression seems to consistently differ

358 between in vivo heart tissues and in vitro differentiation protocols and could therefore be used as a marker to

359 improve in vitro protocols.

4.5 CX43 should be used with caution as a mature cardiomyocyte marker as it is also expressed in the mesoderm and immature cardiomyocytes in the developing heart

363 The mature cardiomyocyte marker, $\mathrm{CX} 43$, was evident in the mesoderm and immature and mature

364 cardiomyocytes in the pig, while it was expressed mostly at early and not later time points in the human 365 differentiating iPSCs. Interestingly, it was downregulated at the pig cardiac progenitor stages but well 366 detectable in the differentiating hiPSCs at D3. Both the pig heart and human iPSC model expressed CX43 at 367 the cardiac mesoderm stage. In contrast, CX43 is first expressed in the mouse heart at E9.5 while being 368 detectable in other tissues much earlier [61-63]. Therefore, our data suggest that CX43 expression differs in 369 the mouse compared to pigs and potentially humans, as suggested by our hiPSC data. However, this would 370 have to be elucidated in detail in future studies.

371 At later stages of human fetal development, CX43 has been shown to be upregulated at the time when fetal 372 cardiomyocytes align in the smooth helical organization needed for coordinated contraction of the ventricle 373 and normal heart function [64]. Hence, it is used in vitro differentiation as a marker for mature and synchronized 
374 cardiomyocytes $[2-4,65,66]$. In our hiPSC differentiation model, no maturation or synchronization step was

375 included and we therefore expected low or absent expression of CX43 in the cardiomyocytes on D7. We

376 suggest that CX43 should only be assessed when comparing expression levels between matured and

377 immature cardiomyocytes, in order to determine the degree of maturation and synchronization. Importantly,

378 the cells should be co-labeled with other stage-specific markers, such as sarcomeric proteins to confirm

379 cardiomyocyte identity.

380

381 5. Conclusion

382 We have for the first time performed a temporal evaluation of commonly used markers for human 383 cardiomyocyte differentiation protocols using a large mammalian model, the pig. We found that expression 384 within the developing pig heart overlapped well with murine and human expression for the mesoderm markers 385 FLK1 and the progenitor marker ISL1. Expression in the pig also faithfully recapitulated expression of the 386 immature cardiomyocyte marker, MYHC-B and the mature cardiomyocyte marker CX43, when compared to 387 the human. However, MYHC-B was also detectable in cardiac progenitor cells, immature cardiomyocytes and 388 ventricular mature cardiomyocytes in both the pig and hiPSC model. Further, expression in the pig heart and 389 the hiPSC model indicated that CX43 is also expressed in mesoderm and immature cardiomyocytes and not 390 only in matured cardiomyocytes. Based on this study, we propose that a combination of FLK1+/PDGFR- $\alpha+$ 391 would be valuable for identifying cardiac mesoderm, ISL1+/NKX2.5+ is a good combination for identifying 392 cardiac progenitors and CTNT+/MYHC-B+ for marking immature cardiomyocytes. CTNT+/ISL1+ could be 393 used to mark immature cardiomyocytes and CTNT+/ISL1- used to mark mature cardiomyocytes. CX43 394 remains an excellent marker for maturation and synchronization of cardiomyocytes, but should be used in 395 combination of other sarcomeric proteins to confirm cardiomyocyte identity. This comparison of the expression 396 of cardiogenesis markers in various species will lead to improved characterization of differentiating human 397 cardiomyocytes in the future.

\section{Acknowledgements}

400 We acknowledge the Core Facility for Integrated Microscopy, Faculty of Health and Medical Sciences, 401 University of Copenhagen for access to and use of the Axio Scan Z.1. We also thank Preben Dybdahl Thomsen 402 for supply of the pig fetuses. 


\section{Author Disclosure Statement}

405 All authors declare that they have no competing interests.

408 Funding statement

409 The project was financed by The Independent Research Fund, Denmark, under the grant (ID: DFF-7017410 00071), The Innovation Foundation (Brainstem (4108-00008B)) and partly by the Danish Environmental 411 Protection Agency as a project under the Centre on Endocrine Disrupters (CEHOS).

\section{References}

414 1. Burridge PW, G Keller, JD Gold and JC Wu (2012). Production of de novo cardiomyocytes: Human pluripotent stem 415 cell differentiation and direct reprogramming. Cell Stem Cell 10: 16-28.

416 2. Kempf H, R Olmer, C Kropp, M R??ckert, M Jara-Avaca, D Robles-Diaz, A Franke, DA Elliott, D Wojciechowski, M

417 Fischer, A Roa Lara, G Kensah, I Gruh, A Haverich, U Martin and R Zweigerdt (2014). Controlling expansion and

418 cardiomyogenic differentiation of human pluripotent stem cells in scalable suspension culture. Stem Cell Reports 3:

419 1132-1146.

420 3. Lundy SD, WZ Zhu, M Regnier and MA Laflamme (2013). Structural and functional maturation of cardiomyocytes 421 derived from human pluripotent stem cells. Stem Cells Dev 22: 1991-2002.

422 4. Marsano A, C Conficconi, M Lemme, P Occhetta, E Gaudiello, E Votta, G Cerino, A Redaelli and M Rasponi (2015).

423 Beating heart on a chip: a novel microfluidic platform to generate functional 3D cardiac microtissues. Lab Chip 16: 599424610.

425 5. Cohen ED, Y Tian and EE Morrisey (2008). Wnt signaling: An essential regulator of cardiovascular differentiation, 426 morphogenesis and progenitor self-renewal. Development 135: 789-798.

427 6. Laugwitz K-L, A Moretti, J Lam, P Gruber, Y Chen, S Woodard, L-Z Lin, C-L Cai, MM Lu, M Reth, O Platoshyn, JX-J 428 Yuan, S Evans and KR Chien (2005). Postnatal isl1+ cardioblasts enter fully differentiated cardiomyocyte lineages.

429 Nature 433: 647-653.

430 7. Martin-Puig S, Z Wang and KR Chien (2008). Lives of a Heart Cell: Tracing the Origins of Cardiac Progenitors. Cell 431 Stem Cell 2: 320-331.

432 8. Martin CM, AP Meeson, SM Robertson, TJ Hawke, JA Richardson, S Bates, SC Goetsch, TD Gallardo and DJ Garry 
433 (2004). Persistent expression of the ATP-binding cassette transporter, Abcg2, identifies cardiac SP cells in the

434 developing and adult heart. Dev Biol 265: 262-275.

435 9. Mummery CL, J Zhang, ES Ng, DA Elliott, AG Elefanty and TJ Kamp (2012). Differentiation of human embryonic stem

436 cells and induced pluripotent stem cells to cardiomyocytes: A methods overview. Circ Res 111: 344-358.

437 10. Oh H, SB Bradfute, TD Gallardo, T Nakamura, V Gaussin, Y Mishina, J Pocius, LH Michael, RR Behringer, DJ Garry,

438 ML Entman and MD Schneider (2003). Cardiac progenitor cells from adult myocardium: Homing, differentiation, and

439 fusion after infarction. Proc Natl Acad Sci U S A 100: 12313-12318.

440 11. Sahara M, F Santoro, J Sohlmér, C Zhou, N Witman, CY Leung, M Mononen, K Bylund, P Gruber and KR Chien

441 (2019). Population and Single-Cell Analysis of Human Cardiogenesis Reveals Unique LGR5 Ventricular Progenitors in

442 Embryonic Outflow Tract. Dev Cell 48: 475-490.e7.

443 12. Synnergren J, C Améen, A Jansson and P Sartipy (2012). Global transcriptional profiling reveals similarities and

444 differences between human stem cell-derived cardiomyocyte clusters and heart tissue. Physiol Genomics 44: $245-258$.

445 13. Cui Y, Y Zheng, X Liu, L Yan, X Fan, J Yong, Y Hu, J Dong, Q Li, X Wu, S Gao, J Li, L Wen, J Qiao and F Tang

446 (2019). Single-Cell Transcriptome Analysis Maps the Developmental Track of the Human Heart. Cell Rep 26: 1934-

447 1950.e5.

448 14. England J and S Loughna (2013). Heavy and light roles: Myosin in the morphogenesis of the heart. Cell Mol Life Sci

449 70: 1221-1239.

450 15. Protze SI, JH Lee and GM Keller (2019). Human Pluripotent Stem Cell-Derived Cardiovascular Cells: From

451 Developmental Biology to Therapeutic Applications. Cell Stem Cell 25: 311-327.

452 16. Kobayashi T, H Zhang, WWC Tang, N Irie, S Withey, D Klisch, A Sybirna, S Dietmann, DA Contreras, R Webb, C

453 Allegrucci, R Alberio and MA Surani (2017). Principles of early human development and germ cell program from

454 conserved model systems. Nature 546: 416-420.

455 17. Rothkötter HJ, E Sowa and R Pabst (2002). The pig as a model of developmental immunology. Hum Exp Toxicol 21:

$456533-536$.

457 18. Butler JE, KM Lager, I Splichal, D Francis, I Kacskovics, M Sinkora, N Wertz, J Sun, Y Zhao, WR Brown, R DeWald, 458 S Dierks, S Muyldermans, JK Lunney, PB McCray, CS Rogers, MJ Welsh, P Navarro, F Klobasa, F Habe and J

459 Ramsoondar (2009). The piglet as a model for B cell and immune system development. Vet Immunol Immunopathol 128:

$460 \quad 147-170$.

461 19. Guilloteau P, R Zabielski, HM Hammon and CC Metges (2010). Nutritional programming of gastrointestinal tract 
development. Is the pig a good model for man? Nutr Res Rev 23: 4-22.

463 20. Buddington RK, PT Sangild, B Hance, EY Huang and DD Black (2012). Prenatal gastrointestinal development in the 464 pig and responses after preterm birth. J Anim Sci 90 Suppl 4: 290-298.

465 21. Conrad MS and RW Johnson (2015). The domestic piglet: an important model for investigating the

466 neurodevelopmental consequences of early life insults. Annu Rev Anim Biosci 3: 245-264.

467 22. Trujano M and AE Wrathall (1985). Observations on the development in vitro of early (13-day) and later (15-day)

468 porcine embryos. Br Vet J 141: 378-387.

469 23. Hyttel P, F Sinowatz, M Vejlsted and K Betteridge (2009). Essentials of domestic animal embryology

470 (Saunders/Elsevier).

471 24. Guerreiro D, SC Lennox and RH Anderson (1980). Postnatal development of the pig heart. Cardiovasc Res 14: 675472679.

473 25. Federspiel JD, P Tandon, CM Wilczewski, L Wasson, LE Herring, SS Venkatesh, IM Cristea and FL Conlon (2019).

474 Conservation and divergence of protein pathways in the vertebrate heart. PLoS Biol 17:.

475 26. Lauschke K, AK Rosenmai, I Meiser, JC Neubauer, K Schmidt, MA Rasmussen, B Holst, C Taxvig, JK Emnéus and 476 AM Vinggaard (2020). A novel human pluripotent stem cell-based assay to predict developmental toxicity. Arch Toxicol.

477 27. Nguyen D (2013). Quantifying chromogen intensity in immunohistochemistry via reciprocal intensity. Protoc Exch 14787.

479 28. Kattman SJ, AD Witty, M Gagliardi, NC Dubois, M Niapour, A Hotta, J Ellis and G Keller (2011). Stage-specific 480 optimization of activin/nodal and BMP signaling promotes cardiac differentiation of mouse and human pluripotent stem 481 cell lines. Cell Stem Cell 8: 228-240.

482 29. Hirata H, S Kawamata, Y Murakami, K Inoue, A Nagahashi, M Tosaka, N Yoshimura, Y Miyamoto, H Iwasaki, T 483 Asahara and Y Sawa (2007). Coexpression of platelet-derived growth factor receptor alpha and fetal liver kinase 1 484 enhances cardiogenic potential in embryonic stem cell differentiation in vitro. J Biosci Bioeng 103: 412-419.

485 30. Kataoka H, N Takakura, S Nishikawa, K Tsuchida, H Kodama, T Kunisada, W Risau, T Kita and SI Nishikawa (1997). 486 Expressions of PDGF receptor alpha, c-Kit and Flk1 genes clustering in mouse chromosome 5 define distinct subsets of 487 nascent mesodermal cells. Dev Growth Differ 39: 729-740.

488 31. Vieira JM, C Ruhrberg and Q Schwarz (2010). VEGF receptor signaling in vertebrate development. Organogenesis 489 6: 97-106.

490 32. Cai CL, X Liang, Y Shi, PH Chu, SL Pfaff, J Chen and S Evans (2003). Isl1 identifies a cardiac progenitor population 
491 that proliferates prior to differentiation and contributes a majority of cells to the heart. Dev Cell.

492 33. Milgrom-Hoffman M, Z Harrelson, N Ferrara, E Zelzer, SM Evans and E Tzahor (2011). The heart endocardium is

493 derived from vascular endothelial progenitors. Development 138: 4777-4787.

494 34. He Z, M Grunewald, Y Dor and E Keshet (2016). VEGF regulates relative allocation of IsI1+ cardiac progenitors to

495 myocardial and endocardial lineages. Mech Dev 142: 40-49.

496 35. Ma Q, B Zhou and WT Pu (2008). Reassessment of IsI1 and Nkx2-5 cardiac fate maps using a Gata4-based reporter

497 of Cre activity. Dev Biol 323: 98-104.

498 36. Karlsson O, S Thor, T Norberg, H Ohlsson and T Edlund (1990). Insulin gene enhancer binding protein Isl-1 is a

499 member of a novel class of proteins containing both a homeo-and a Cys-His domain. Nature 344: 879-882.

500 37. Pfaff SL, M Mendelsohn, CL Stewart, T Edlund and TM Jessell (1996). Requirement for LIM homeobox gene Isl1 in

501 motor neuron generation reveals a motor neuron-dependent step in interneuron differentiation. Cell 84: 309-320.

502 38. Ahlgren U, SL Pfaff, TM Jessell, T Edlund and H Edlund (1997). Independent requirement for ISL1 in formation of

503 pancreatic mesenchyme and islet cells. Nature 385: 257-260.

504 39. Yuan S and GC Schoenwolf (2000). Islet-1 marks the early heart rudiments and is asymmetrically expressed during 505 early rotation of the foregut in the chick embryo. Anat Rec 260: 204-207.

506 40. Radde-Gallwitz K, L Pan, L Gan, X Lin, N Segil and P Chen (2004). Expression of Islet1 marks the sensory and

507 neuronal lineages in the mammalian inner ear. J Comp Neurol 477: 412-421.

508 41. Elshatory $Y$ and L Gan (2008). The LIM-homeobox gene Islet-1 is required for the development of restricted forebrain 509 cholinergic neurons. J Neurosci 28: 3291-3297.

510 42. Elshatory Y, D Everhart, M Deng, X Xie, RB Barlow and L Gan (2007). Islet-1 controls the differentiation of retinal 511 bipolar and cholinergic amacrine cells. J Neurosci 27: 12707-12720.

512 43. Nathan E, A Monovich, L Tirosh-Finkel, Z Harrelson, T Rousso, A Rinon, I Harel, SM Evans and E Tzahor (2008).

513 The contribution of Islet1-expressing splanchnic mesoderm cells to distinct branchiomeric muscles reveals significant

514 heterogeneity in head muscle development. Development 135: 647-657.

515 44. Sun Y, IM Dykes, X Liang, SR Eng, SM Evans and EE Turner (2008). A central role for Islet1 in sensory neuron

516 development linking sensory and spinal gene regulatory programs. Nat Neurosci 11: 1283-1293.

517 45. Westerlund J, L Andersson, T Carlsson, P Zoppoli, H Fagman and M Nilsson (2008). Expression of islet1 in thyroid

518 development related to budding, migration, and fusion of primordia. Dev Dyn 237: 3820-3829. 

Gene Expr Patterns 13: 407-412.

521 47. Lien CL, C Wu, B Mercer, R Webb, JA Richardson and EN Olson (1999). Control of early cardiac-specific

522 transcription of Nkx2-5 by a GATA-dependent enhancer. Development 126: 75-84.

523 48. Lints TJ, LM Parsons, L Hartley, I Lyons and RP Harvey (1993). Nkx-2.5: a novel murine homeobox gene expressed

524 in early heart progenitor cells and their myogenic descendants. Development 119: 419-431.

525 49. Yang L, MH Soonpaa, ED Adler, TK Roepke, SJ Kattman, M Kennedy, E Henckaerts, K Bonham, GW Abbott, RM Linden, LJ Field and GM Keller (2008). Human cardiovascular progenitor cells develop from a KDR+ embryonic-stemcell-derived population. Nature 453: 524-528. 50. Elliott DA, SR Braam, K Koutsis, ES Ng, R Jenny, EL Lagerqvist, C Biben, T Hatzistavrou, CE Hirst, QC Yu, RJP Skelton, D Ward-van Oostwaard, SM Lim, O Khammy, X Li, SM Hawes, RP Davis, AL Goulburn, R Passier, OWJ Prall, JM Haynes, CW Pouton, DM Kaye, CL Mummery, AG Elefanty and EG Stanley (2011). NKX2-5(eGFP/w) hESCs for isolation of human cardiac progenitors and cardiomyocytes. Nat Methods 8: 1037-1040.

532 51. Wang Q, RS Reiter, Q-Q Huang, J-P Jin and JJ-C Lin (2001). Comparative studies on the expression patterns of 533 threetroponin T genes during mouse development. Anat Rec 263: 72-84.

534 52. Fischer B, A Meier, A Dehne, A Salhotra, TA Tran, S Neumann, K Schmidt, I Meiser, JC Neubauer, H Zimmermann 535 and L Gentile (2018). A complete workflow for the differentiation and the dissociation of hiPSC-derived cardiospheres. 536 Stem Cell Res 32: 65-72.

537 53. Velasquez-Mao AJ, CJM Tsao, MN Monroe, X Legras, B Bissig-Choisat, KD Bissig, R Ruano and JG Jacot (2017). 538 Differentiation of spontaneously contracting cardiomyocytes from non-virally reprogrammed human amniotic fluid stem cells. PLoS One 12: 1-12.

540 54. Jin Y, Y Liu, Z Li, K Santostefano, J Shi, X Zhang, D Wu, Z Cheng, W Wu, N Terada, S Jin and F Bai (2018).

541 Enhanced differentiation of human pluripotent stem cells into cardiomyocytes by bacteria-mediated transcription factors

542 delivery. PLoS One 13: 1-14.

543 55. Tsao CJ, F Taraballi, L Pandolfi, AJ Velasquez-Mao, R Ruano, E Tasciotti and JG Jacot (2018). Controlled release of 544 small molecules for cardiac differentiation of pluripotent stem cells. Tissue Eng - Part A 24: 1798-1807.

545 56. Kay M, BM Soltani, FH Aghdaei, H Ansari and H Baharvand (2019). Hsa-miR-335 regulates cardiac mesoderm and 546 progenitor cell differentiation. Stem Cell Res Ther 10: 1-13.

547 57. Burridge PW, E Matsa, P Shukla, ZC Lin, JM Churko, AD Ebert, F Lan, S Diecke, B Huber, NM Mordwinkin, JR 
549 Methods 11: 855-860.

550 58. Everett AW (1986). Isomyosin expression in human heart in early pre- and post-natal life. J Mol Cell Cardiol 18: 607615.

552 59. Lyons GE, S Schiaffino, D Sassoon, P Barton and M Buckingham (1990). Developmental regulation of myosin gene 553 expression in mouse cardiac muscle. J Cell Biol 111: 2427-2436.

554 60. Friedman CE, Q Nguyen, SW Lukowski, A Helfer, HS Chiu, J Miklas, S Levy, S Suo, JDJ Han, P Osteil, G Peng, N 555 Jing, GJ Baillie, A Senabouth, AN Christ, TJ Bruxner, CE Murry, ES Wong, J Ding, Y Wang, J Hudson, H RuoholaBaker, Z Bar-Joseph, PPL Tam, JE Powell and NJ Palpant (2018). Single-Cell Transcriptomic Analysis of Cardiac Differentiation from Human PSCs Reveals HOPX-Dependent Cardiomyocyte Maturation. Cell Stem Cell 23: 586-598.e8. 61. Delorme B, E Dahl, T Jarry-Guichard, JP Briand, K Willecke, D Gros and M Théveniau-Ruissy (1997). Expression pattern of connexin gene products at the early developmental stages of the mouse cardiovascular system. Circ Res 81: 423-437.

62. Ruangvoravat CP and CW Lo (1992). Connexin 43 expression in the mouse embryo: Localization of transcripts within developmentally significant domains. Dev Dyn 194: 261-281. connexin43 during mouse gastrulation and organogenesis. Development 114: 203-212. of connexins in the human fetal heart. Sci Rep 7: 1-9.

567 65. Duan Y, Z Liu, J O’Neill, LQ Wan, DO Freytes and G Vunjak-Novakovic (2011). Hybrid gel composed of native heart matrix and collagen induces cardiac differentiation of human embryonic stem cells without supplemental growth factors. J Cardiovasc TransI Res 4: 605-615. Lyons, JA Thomson, TJ Herron, J Jalife and TJ Kamp (2012). Extracellular matrix promotes highly efficient cardiac

572 differentiation of human pluripotent stem cells: The matrix sandwich method. Circ Res 111: 1125-1136.

\section{4 corresponding author}

575

576 Karin Lauschke 
577 National Food Institute

578 Technical University of Denmark

579 Kemitorvet 202

5802800 Kongens. Lyngby

581 karla@food.dtu.dk 
A

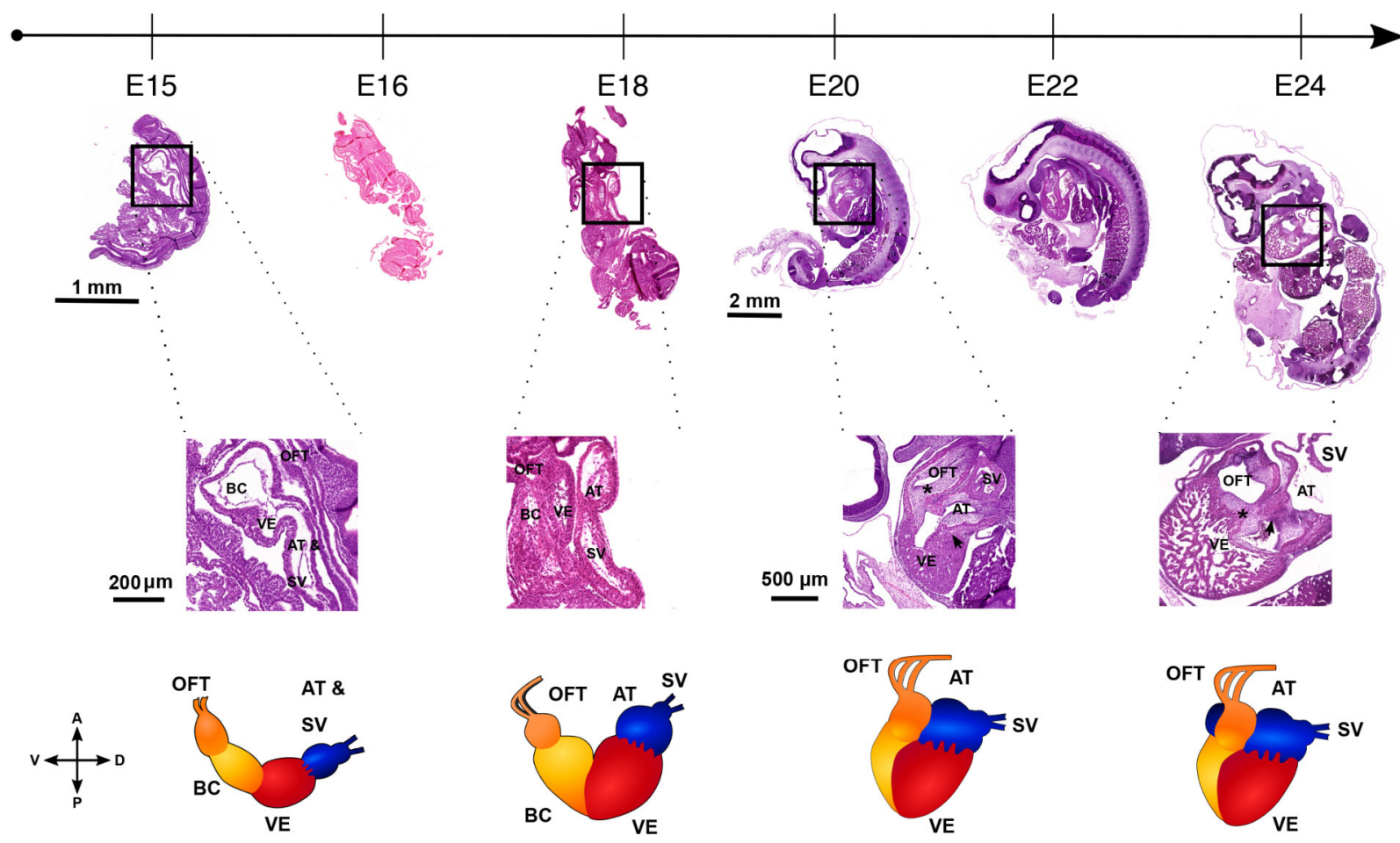

B

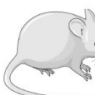

E8.0 - Linear tube

E9.0 - Looping heart

E9.5 - ECC formation

E11.5 - OFT division

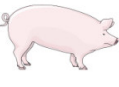

E15/16 - Linear tube

E18 - Looping heart

E20/22 - ECC formation

E24 - OFT division

E21 - Linear tube

E28 - Looping heart

E35 - ECC formation

E42 - OFT division

Figure 1: Pig heart developmental stages. A) Sagittal sections of HE-stained pig embryos at indicated days of embryonic development (E15 - E24) (upper panel) and magnifications of the heart area with depicted heart structures (lower panel).VE: Ventricle, OFT: Outflow tract, AT: Atrium, BC: Bulbus cordis, SV: Sinus venosus. *: conu-truncal cushions, little black arrow: endocardial cushions. Orientation arrows: A: Anterior, P: Posterior, D: Dorsal, V: Ventral. B) Table indicating the stages in embryonic days and key morphological features compared in the mouse, pig and human. ECC: Endocardial cushions. 

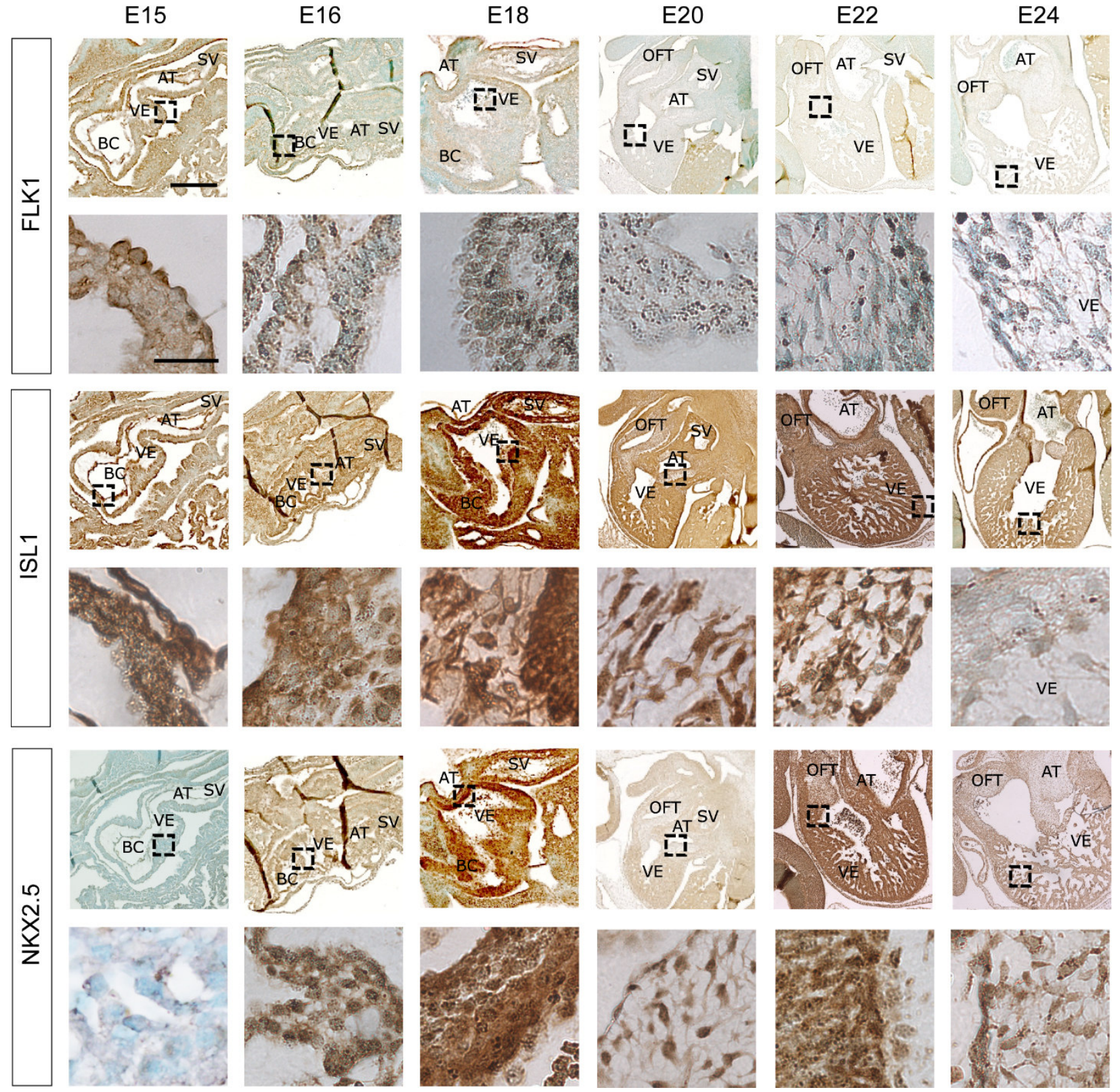

Figure 2: Expression of early cardiac markers in the pig heart. A) Immunohistochemical images of

FLK1, ISL1 and NKX2.5 in the pig heart at embryonic days E15 - 24 with overview images of the heart (upper row) and magnifications to see cellular distribution (lower row). Representative images of sections from three embryos per marker. VE: Ventricle, OFT: Outflow tract, AT: Atrium, BC: Bulbus cordis, SV: Sinus venosus. Scale bar for low magnification $=200 \mu \mathrm{M}$, higher magnification images $=30 \mu \mathrm{M}$. 


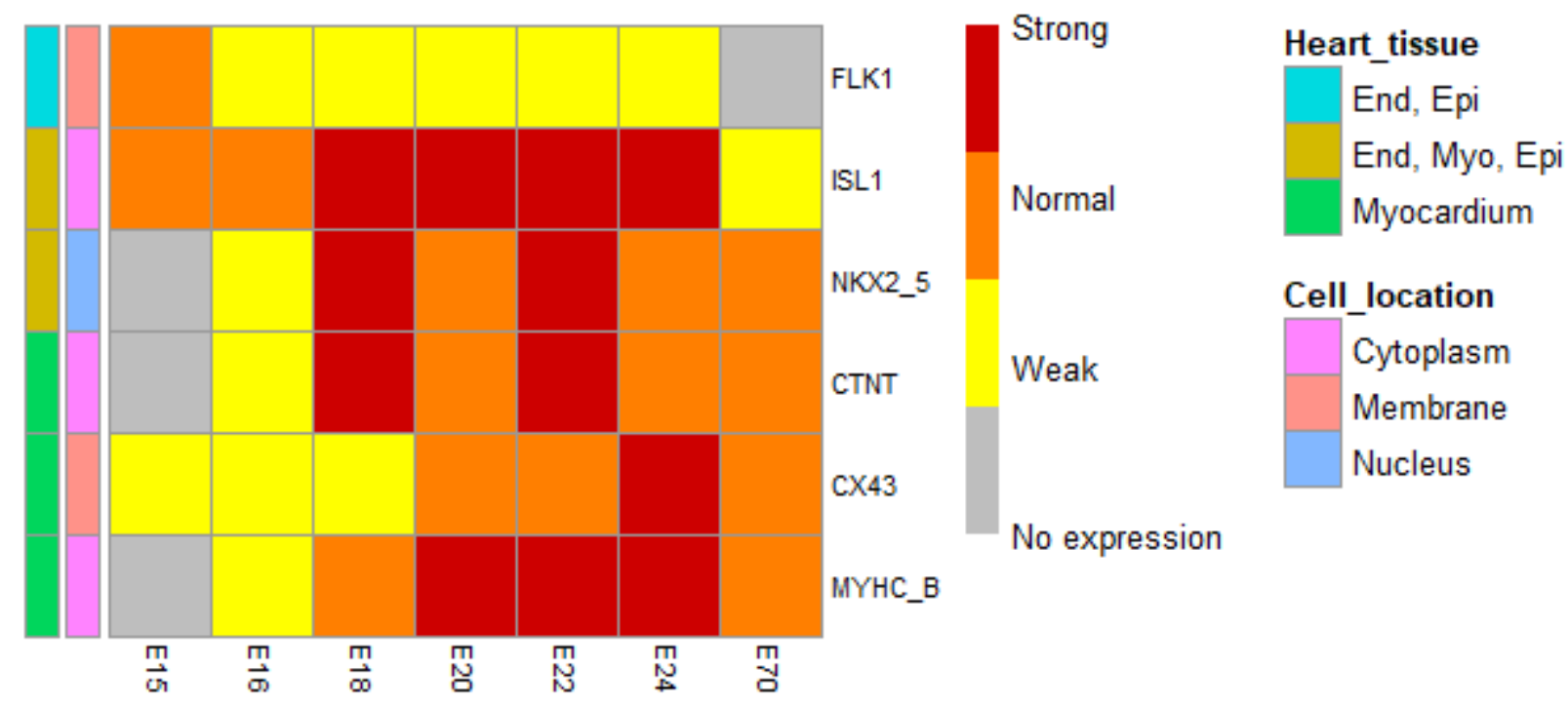

Figure 3: Marker expression levels during pig embryonic heart development. Reciprocal intensity values from immunohistochemical images displaying strong, normal, weak or no expression. Predominant location of each marker is indicated (Heart tissue as well as cellular location). Average values of all slices from three pig embryos. 
A
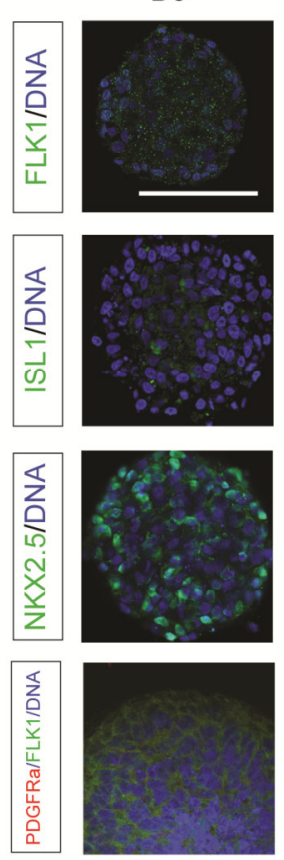

B
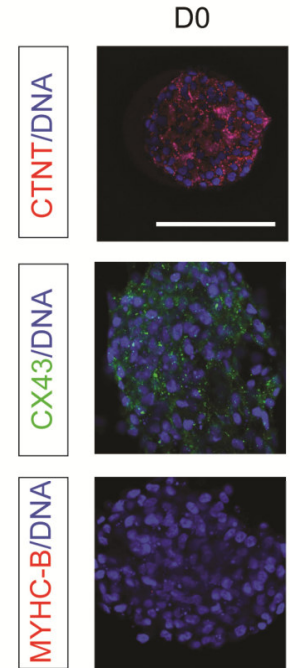

D1
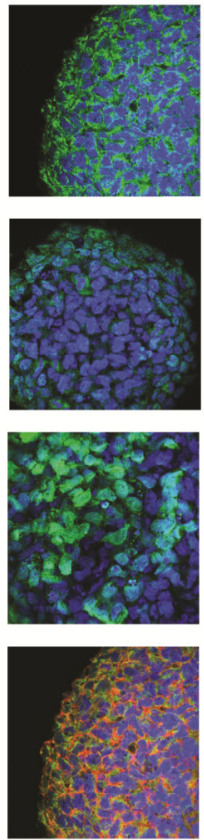

D1
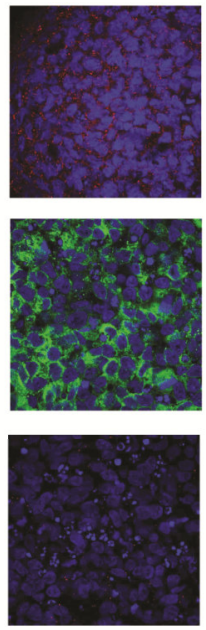

D2
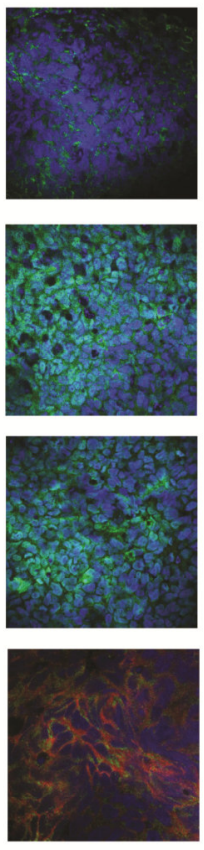

D2
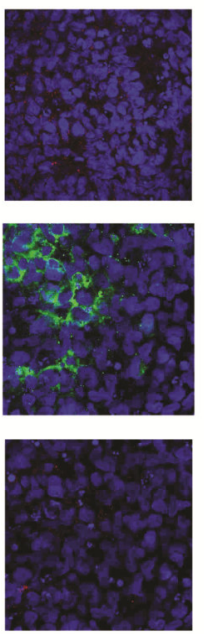

D3
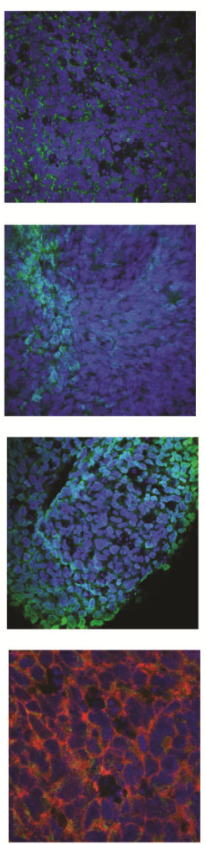

D3
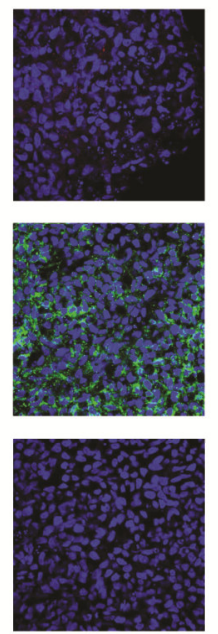

D6
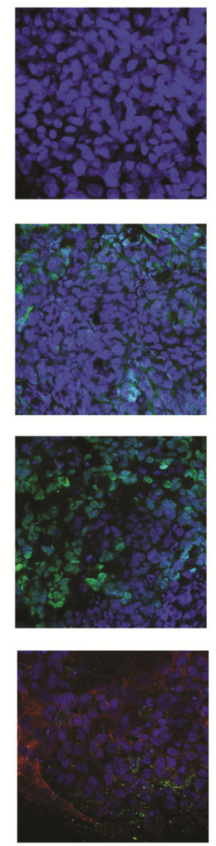

D6
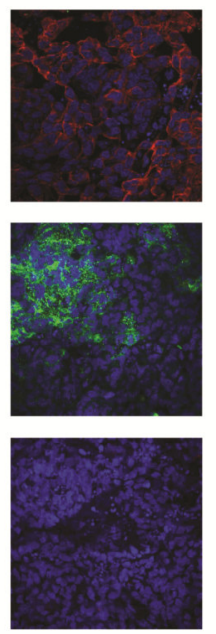

D7
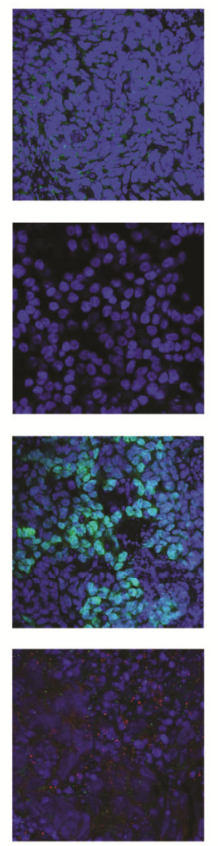

D7
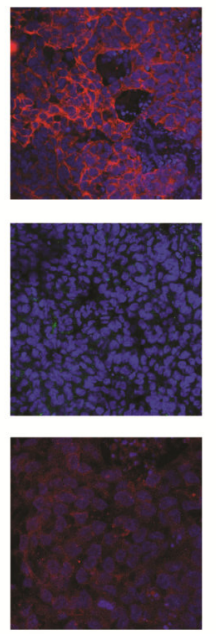

Figure 4: Expression of cardiac markers in the human cardiomyocyte model. A) Immunohistochemical images of early cardiac markers FLK1, ISL1, NKX2.5 and PDGFR- $\alpha$ in differentiated human iPSCs from day D0 - D7 of differentiation. B) Immunohistochemical images of late cardiac markers CTNT, CX43 and MYHC-B in differentiated human iPSCs from day D0 - D7 of differentiation. Counterstaining with Hoechst (blue) for DNA. Representative images of 2 - 3 embryoid bodies per marker. Scale bar $=500 \mathrm{um}$. 

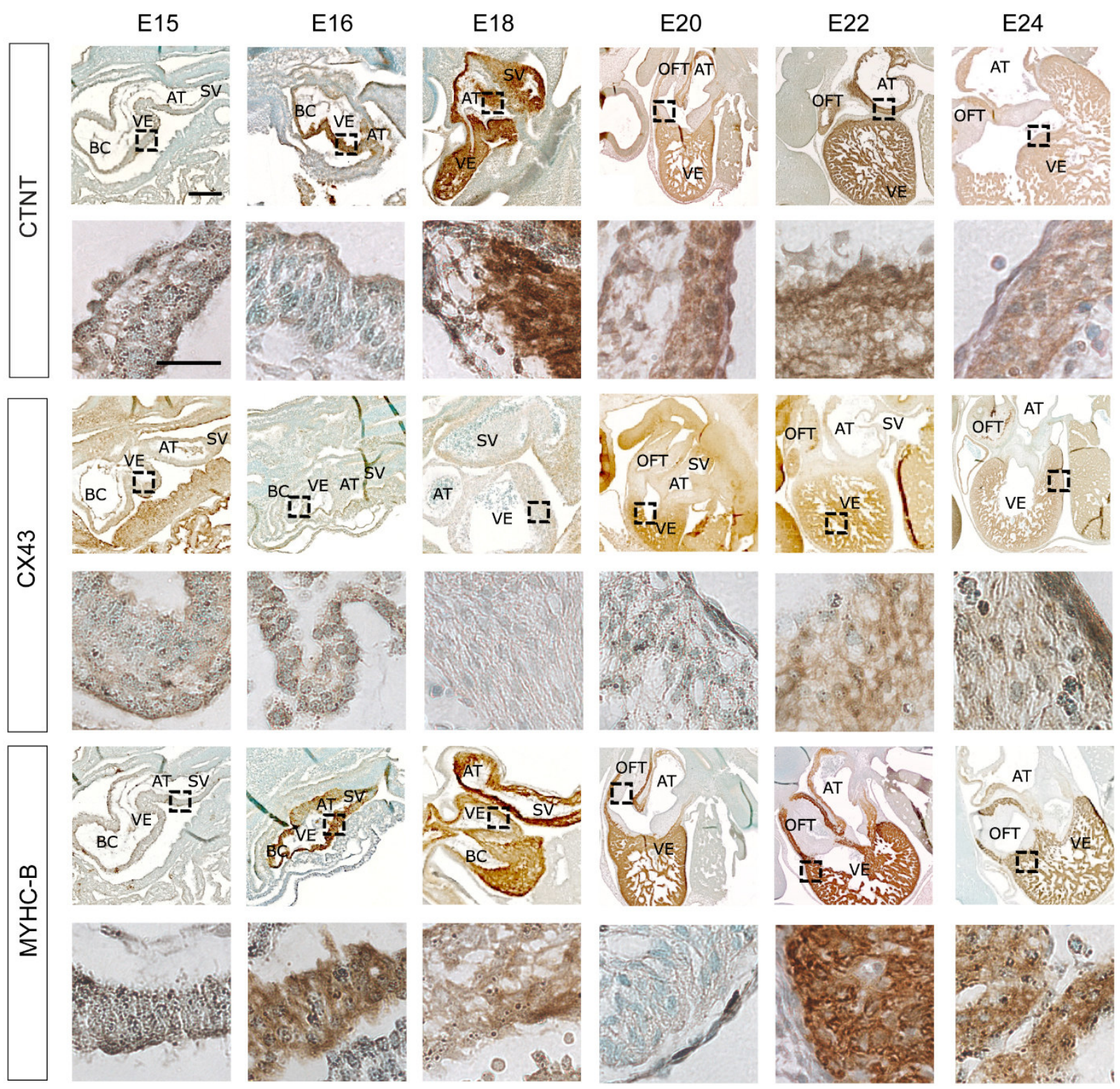

Figure 5: Expression of late cardiac markers in the pig heart. A) Immunohistochemical images of CTNT, CX43 and MYHC-B in the pig heart at embryonic days E15 - 24 with overview images of the heart (upper row) and magnifications to see cellular distribution (lower row). Representative images of sections from three embryos per marker. VE: Ventricle, OFT: Outflow tract, AT: Atrium, BC: Bulbus cordis, SV: Sinus venosus. Scale bar for low magnification $=200 \mu \mathrm{M}$, higher magnification images $=30 \mu \mathrm{M}$. 


\section{Supplementary material}

Table 1: Cardiomyocyte differentiation media

\begin{tabular}{|c|c|}
\hline \multicolumn{2}{|l|}{ mTeSR-ROCK } \\
\hline mTeSR1 (STEMCELL Technologies 85850) & $10 \mathrm{ml}$ \\
\hline ROCK (5 mM, Cayman Chemical 10005583) & $20 \mu \mathrm{l}$ \\
\hline PSG (Life technologies 10378016) & $100 \mu \mathrm{l}$ \\
\hline \multicolumn{2}{|l|}{ DO medium } \\
\hline KO-DMEM & $10 \mathrm{ml}$ \\
\hline PSG (Life technologies 10378016) & $100 \mu \mathrm{l}$ \\
\hline ITS (BD 354351) & $10 \mu \mathrm{l}$ \\
\hline Y (10 mM, Abcam 120129) & $10 \mu \mathrm{l}$ \\
\hline FGF2 (10 $\mu \mathrm{g} / \mathrm{ml}$, Peprotech 100-18B) & $10 \mu \mathrm{l}$ \\
\hline Activin A (10 $\mu \mathrm{g} / \mathrm{ml}$, eBioscience 34-8993-85) & $10 \mu \mathrm{l}$ \\
\hline CHIR (1 mM, Axon Medchem 1386) & $25 \mu \mathrm{l}$ \\
\hline BMP4 (10 $\mu \mathrm{g} / \mathrm{ml}, \mathrm{R} \& \mathrm{D}$ 314-BP-010) & $1 \mu \mathrm{l}$ \\
\hline \multicolumn{2}{|l|}{ TS medium } \\
\hline KO-DMEM (Life technologies 10829018) & $10 \mathrm{ml}$ \\
\hline PSG (Life technologies 10378016) & $100 \mu \mathrm{l}$ \\
\hline TS * & $100 \mu \mathrm{l}$ \\
\hline Asc (250 mM, Sigma-Aldrich 49752) & $10 \mu \mathrm{l}$ \\
\hline \multicolumn{2}{|l|}{ Wnt medium } \\
\hline KO-DMEM (Life technologies 10829018) & $10 \mathrm{ml}$ \\
\hline PSG (Life technologies 10378016) & $100 \mu \mathrm{l}$ \\
\hline TS * & $100 \mu \mathrm{l}$ \\
\hline Asc (250 mM, Sigma-Aldrich 49752) & $10 \mu \mathrm{l}$ \\
\hline Wnt (2mM, Tocris Cat.-No. 5148) & $20 \mu \mathrm{l}$ \\
\hline
\end{tabular}

${ }^{*} \mathrm{TS}$ is prepared by adding $1 \mathrm{ml}$ of sodium selenite $(27 \mathrm{mg}$ sodium selenite Sigma-Aldrich S5261 in $400 \mathrm{ml}$ PBS w/o Ca and Mg) to the $99 \mathrm{ml}$ transferrin (55 mg transferrin Sigma-Aldrich T8158 in $99 \mathrm{ml} \mathrm{PBS}$ w/o Ca and $\mathrm{Mg}$ )

Table 2. Reciprocal intensity values with their corresponding integer number used to create the heat map and the heat map label.

\begin{tabular}{|l|c|l|}
\hline Reciprocal intensity values & Integer number & Heat map label \\
\hline $0-50$ & 0 & No expression \\
\hline $50-120$ & 2 & Weak expression \\
\hline $120-170$ & 4 & Normal expression \\
\hline $170-250$ & 6 & Strong expression \\
\hline
\end{tabular}


SSEA3
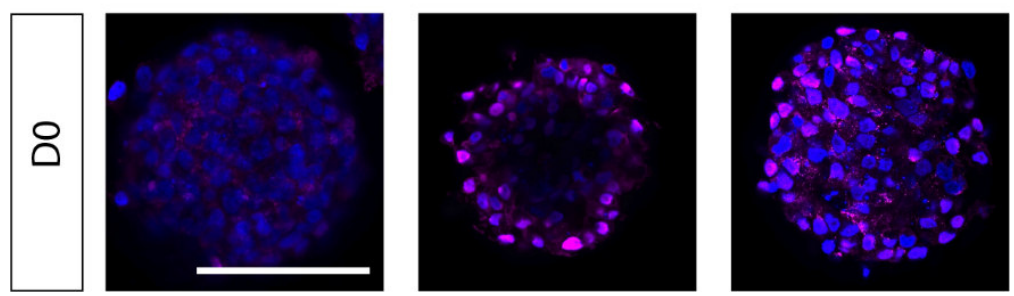

Supplementary figure 1: Pluripotency markers in D0 EBs. Immunohistochemical images of pluripotency markers SSEA3, OCT4 and SOX2 in EBs on D0 of the differentiation protocol.

FLK1
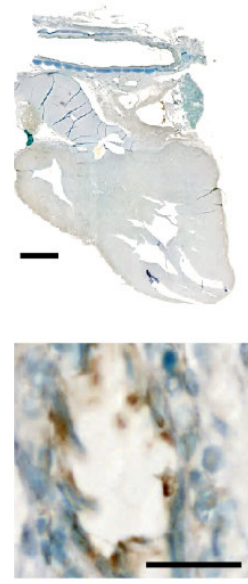

ISL1
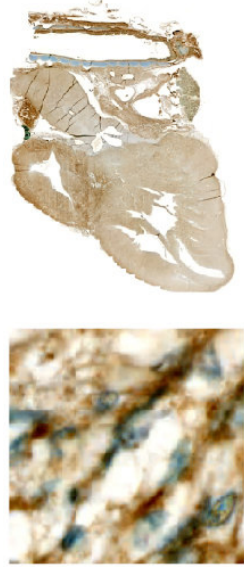

NKX2.5
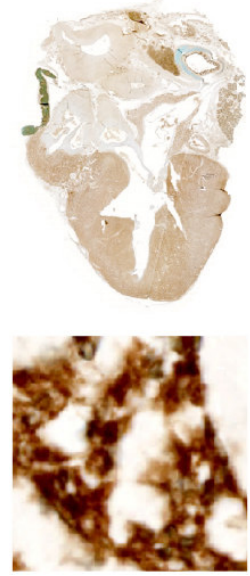

CTNT
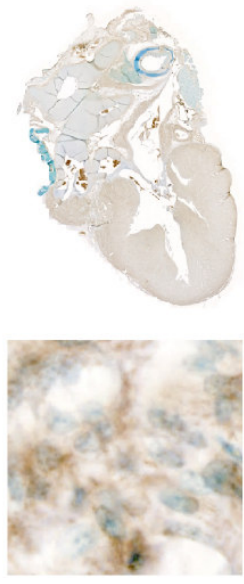

CX43
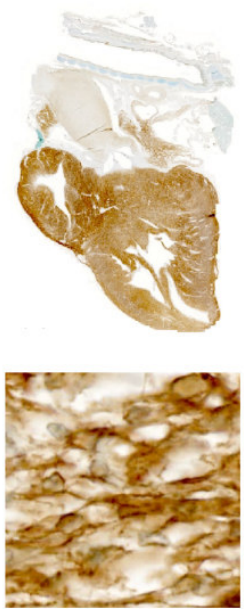

MYHC-B
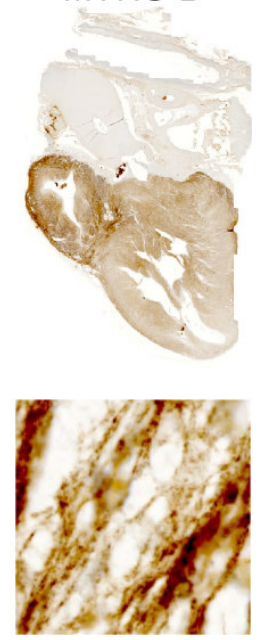

Supplementary figure 2: Marker expression in the E70 pig embryo heart. Immunohistochemical images of FLK1, ISL1, NKX2.5, CTNT, CX43 and MYHC-B in the pig heart at embryonic day E70 with overview images of the heart (upper row) and magnifications to see cellular distribution (lower row). Scale bar for low magnification $=200 \mu \mathrm{M}$, higher magnification images $=30 \mu \mathrm{M}$. 
$\begin{array}{lllll}\text { E16 } & \text { E18 } & \text { E20 } & \text { E22 } & \text { E24 }\end{array}$
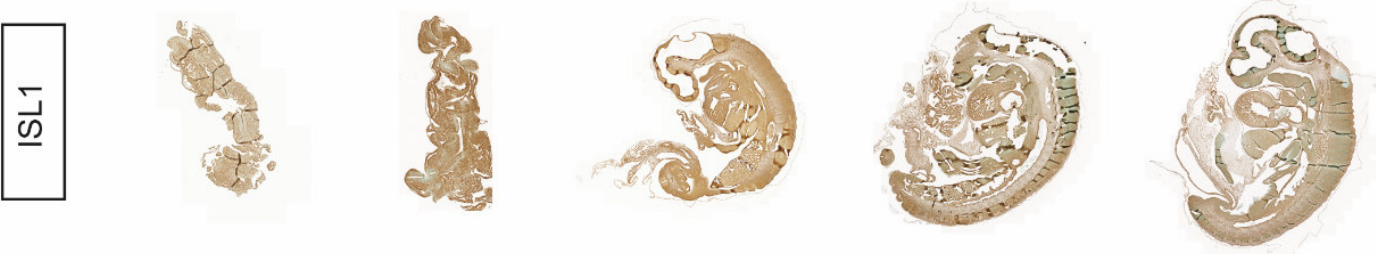

Supplementary figure 3: Overview images of the entire embryo at stages E16 - E24. Immunohistochemical images for IsI1.

A
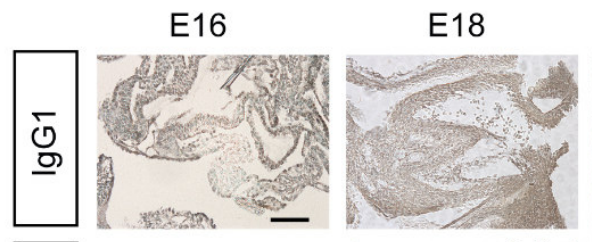

E20

E22

E24

E70
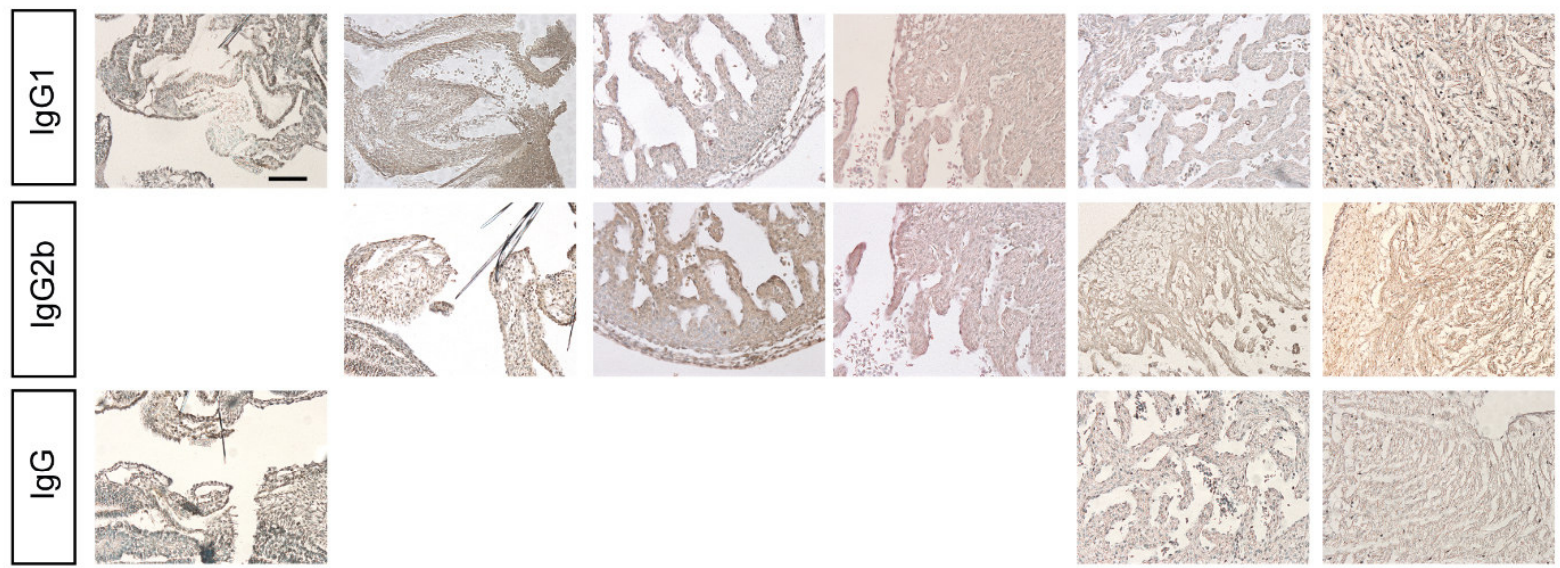

B

Donkey anti-mouse

Donkey anti-rabbit

Donkey anti-goat
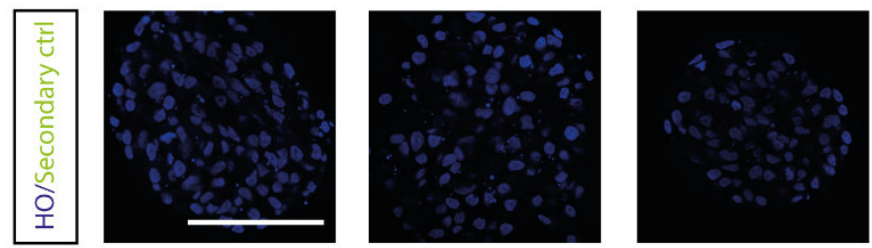

Supplementary figure 4: Negative controls of antibody stainings. A) For immunohistochemistry stainings of pig embryos at indicated stages. The following antibody controls were used: Negative mouse $\lg \mathrm{G} 1$, negative mouse $\lg \mathrm{G} 2 \mathrm{~b}$ and normal rabbit $\lg \mathrm{G}$. B) For immunocytochemistry stainings of D0 EBs, only secondary antibodies were used. 